\title{
Asymmetric Synthesis of Aziridines and Arylalanine Derivatives
}

\author{
Seung-Han Lee* and In-Woong Song \\ Department of Chemistry, Kyunghee University, Yongin 449-701, Korea. ${ }^{*}$ E-mail: shlee@khu.ac.kr \\ Received November 8, 2004
}

Key Words : Aziridines, Arylalanines, Asymmetric synthesis, Cobalt catalyst

Aziridines are useful intermediates for the synthesis of the amino alcohols, amino acids, and nitrogen-containing heterocycles. Though a variety of methods have been developed for the synthesis of aziridines, the simplest one is either the addition of a carbene to an imine or the addition of nitrene to an alkene. ${ }^{1}$ Recent advances have been made in this area using various Lewis acid catalysts including $\mathrm{LiClO}_{4},{ }^{2}$ chiral boron complexes, ${ }^{3} \mathrm{BF}_{3} \cdot \mathrm{Et}_{2} \mathrm{O},{ }^{4} \mathrm{AlCl}_{3},{ }^{4} \mathrm{TiCl}_{4},{ }^{4}$ $\mathrm{SnCl}_{4},{ }^{5} \mathrm{InCl}_{3},{ }^{6} \mathrm{Cu}$ complexes, ${ }^{7} \mathrm{Zn}(\mathrm{OTf})_{2},{ }^{8} \mathrm{Fe}$ complex, $[\operatorname{Ir}(\operatorname{cod}) \mathrm{Cl}]_{2},{ }^{10}$ and $\operatorname{Ln}(\mathrm{OTf})_{3}{ }^{8,11}$ In this report, we wish to describe the asymmetric synthesis of cis-aziridines from chiral $N$-benzylimines and ethyl diazoacetate (EDA) using cobalt(II) as Lewis acid catalyst and the synthesis of arylalanine derivatives by regioselective ring opening of chiral aziridines. We previously reported the aziridine forming reaction from $N$-arylimines and EDA using copper catalysts. $^{7 \mathrm{~b}}$ However, copper catalyzed aziridination did not proceed for $N$-alkylimines. We found the cobalt catalyst gave the $c i s$-aziridines in moderate yields for $N$-alkylimines (Scheme 1 and Table 1). Also, some rearranged products, enamines 4 and 5 were obtained. ${ }^{11 \mathrm{~b}}$

A number of experiments were performed to maximize the efficiency of the aziridine forming reaction. In this effort, we found that reactions of imines with 3 equivalents of EDA using $20 \% \mathrm{CoCl}_{2}$ in the presence of $\mathrm{AgBF}_{4}$ at room temperature gave the aziridines in highest yield (Table 1, entries 1-4). ${ }^{12}$ And the reaction gave the lower yield of aziridine (29\%) and higher yield of rearranged enamines 4 and $5(69 \%)$ in refluxing acetone (entry 10). Though the reaction performed at $0{ }^{\circ} \mathrm{C}$ to improve the diastereoselectivity, the reaction did not proceed (entry 11).<smiles>[R]C(N/C(=C/Br)C(=O)OCC)c1ccccc1</smiles>

4<smiles>[R]C(N/C([Al])=C\COCC)Pc1ccccc1</smiles>

5<smiles>[R]C(N=C[Al])[Pb]=[Fe+]</smiles>

While imines with electron-withdrawing substituent at carbon atom afforded higher yields of the aziridines (entries 13-14), p-methoxybenzaldimine $\mathbf{1 k}$ with electron-releasing substituent at carbon atom gave no reaction (entry 16). The ratios of two cis-aziridines produced in these reactions were found to vary from 1.3 to 2.7. The structure of aziridines was determined by ${ }^{1} \mathrm{H},{ }^{13} \mathrm{C} \mathrm{NMR}$, and elemental analysis. The ${ }^{1} \mathrm{H}$ NMR spectra of the cis-diastereomer of aziridine $\mathbf{2 a}$, prepared from $N$-benzylbenzaldimine, contained two doublets, $2.63(1 \mathrm{H}, J=6.9 \mathrm{~Hz})$ and $3.06(1 \mathrm{H}, J=6.9 \mathrm{~Hz})$

Table 1. Aziridine forming reaction using Co(II) catalyst

\begin{tabular}{cccccc}
\hline Entry & Imine & $\mathrm{R}$ & $\mathrm{Ar}$ & $\mathbf{2 + 3}(\%)^{a}$ & $\mathbf{2 / 3}$ \\
\hline $1^{c}$ & $\mathbf{1 a}$ & $\mathrm{H}$ & $\mathrm{Ph}$ & 8 & - \\
$2^{d}$ & $\mathbf{1 a}$ & $\mathrm{H}$ & $\mathrm{Ph}$ & 20 & - \\
$3^{e}$ & $\mathbf{1 a}$ & $\mathrm{H}$ & $\mathrm{Ph}$ & 34 & - \\
$4^{f}$ & $\mathbf{1 a}$ & $\mathrm{H}$ & $\mathrm{Ph}$ & 45 & - \\
5 & $\mathbf{1 b}$ & $\mathrm{Me}$ & $\mathrm{Ph}$ & 64 & 2.1 \\
6 & $\mathbf{1 c}$ & $\mathrm{Me}$ & $p$ - $\mathrm{FC}_{6} \mathrm{H}_{4}$ & 66 & 2.2 \\
7 & $\mathbf{1 d}$ & $\mathrm{Me}$ & $o-\mathrm{ClC}_{6} \mathrm{H}_{4}$ & 65 & 2.2 \\
8 & $\mathbf{1 e}$ & $\mathrm{Me}$ & $m-\mathrm{ClC}_{6} \mathrm{H}_{4}$ & 71 & 2.4 \\
9 & $\mathbf{1 f}$ & $\mathrm{Me}$ & $p-\mathrm{ClC}_{6} \mathrm{H}_{4}$ & 69 & 2.1 \\
$10^{g}$ & $\mathbf{1 f}$ & $\mathrm{Me}$ & $p-\mathrm{ClC}_{6} \mathrm{H}_{4}$ & 29 & 2.0 \\
$11^{h}$ & $\mathbf{1 f}$ & $\mathrm{Me}$ & $p-\mathrm{ClC}_{6} \mathrm{H}_{4}$ & no reaction & - \\
12 & $\mathbf{1 g}$ & $\mathrm{Me}$ & $p-\mathrm{BrC}_{6} \mathrm{H}_{4}$ & 70 & 2.0 \\
13 & $\mathbf{1 h}$ & $\mathrm{Me}$ & $p-\mathrm{NO}_{2} \mathrm{C}_{6} \mathrm{H}_{4}$ & 89 & 2.7 \\
14 & $\mathbf{1 i}$ & $\mathrm{Me}$ & $p-\mathrm{CNC}_{6} \mathrm{H}_{4}$ & 85 & 2.3 \\
15 & $\mathbf{1 j}$ & $\mathrm{Me}$ & $p-\mathrm{CH}_{3} \mathrm{C}_{6} \mathrm{H}_{4}$ & 43 & 2.4 \\
16 & $\mathbf{1 k}$ & $\mathrm{Me}$ & $p-\mathrm{CH}_{3} \mathrm{OC}_{6} \mathrm{H}_{4}$ & no reaction & - \\
17 & $\mathbf{1 l}$ & $\mathrm{Me}$ & $2-$ naphthyl & 58 & 1.3 \\
18 & $\mathbf{1 m}$ & $\mathrm{Me}$ & $2-\mathrm{pyridyl}$ & 34 & 1.8 \\
\hline
\end{tabular}

$\overline{{ }^{a} \text { Isolated yields. }{ }^{b} \text { Ratios were determined by }{ }^{1} \mathrm{H} \text { NMR analysis. }{ }^{c} \text { One }}$ equivalent of EDA and $10 \% \mathrm{CoCl}_{2}$ were used. ${ }^{d}$ One equivalent of EDA and $20 \% \mathrm{CoCl}_{2}$ were used. ${ }^{e}$ Two equivalents of EDA and $20 \% \mathrm{CoCl}_{2}$ were used. ${ }^{f}$ Three equivalents of EDA and $20 \% \mathrm{CoCl}_{2}$ were used. ${ }^{g}$ The reaction performed in refluxing acetone. ${ }^{h}$ The reaction performed at 0 ${ }^{\circ} \mathrm{C}$.<smiles>[R]C(c1ccccc1)N1[C@H]([13CH])[C@@H]1COCC</smiles>

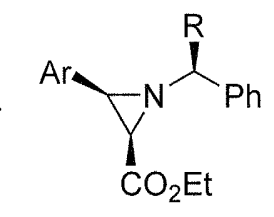

3a-m 
<smiles>CCO[C@H]1[C@@H](C)N1C(C)c1ccccc1</smiles>

2

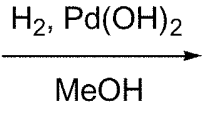<smiles>CCOC(=O)C(N)C[Al]</smiles>

6
Scheme 2

Table 2. Regioselective ring reduction of aziridines $\mathbf{2}$

\begin{tabular}{cccc}
\hline Entry & Aziridine & $\mathrm{Ar}$ & ${\text { Yield }(\%)^{a}}^{a}$ \\
\hline 1 & $\mathbf{2 b}$ & $\mathrm{Ph}$ & 89 \\
2 & $\mathbf{2 f}$ & $p-\mathrm{ClC}_{6} \mathrm{H}_{4}$ & $97^{b}$ \\
3 & $\mathbf{2 g}$ & $p-\mathrm{BrC}_{6} \mathrm{H}_{4}$ & $99^{b}$ \\
4 & $\mathbf{2 h}$ & $p-\mathrm{NO}_{2} \mathrm{C}_{6} \mathrm{H}_{4}$ & $67^{c}$ \\
5 & $\mathbf{2 i}$ & $p-\mathrm{CNC}_{6} \mathrm{H}_{4}$ & 84 \\
6 & $\mathbf{2 l}$ & 2-naphthyl & 94 \\
\hline
\end{tabular}

${ }^{a}$ Isolated yields. ${ }^{b}$ Product was dehalogenated phenylalanine ethyl ester $(\mathrm{Ar}=\mathrm{Ph}) .{ }^{c}$ Product was reduced $p$-aminophenylalanine ethyl ester $(\mathrm{Ar}=$ $\left.p-\mathrm{NH}_{2} \mathrm{C}_{6} \mathrm{H}_{4}\right)$.

ppm corresponding to the vicinally disposed ring protons. ${ }^{11 \mathrm{~b}}$

The absolute stereochemistry of the products $\mathbf{2}$ and $\mathbf{3}$ were determined by transformation of the aziridines to arylalanine derivatives 6 via regioselective reduction of $\mathrm{N}-\mathrm{C} 3$ bond of aziridines using $\mathrm{Pd} / \mathrm{C}$ or $\mathrm{Pd}(\mathrm{OH})_{2} / \mathrm{C}$ (Scheme 2 and Table $2)^{13}$ and by comparison of specific rotations of 6 with those of known compounds. Under the present reaction conditions, ring substituted halogens were reduced to give the phenylalanine ethyl ester (Table 2, entries 2-3) and nitro group was also reduced to give the aminophenylalanine derivative (Table 2, entry 4).

Also, $\mathrm{N}$-Boc protected arylalanines 7 were prepared in high yields by regioselective ring opening of aziridines 2 using catalytic hydrogenolysis in the presence of $(\mathrm{Boc})_{2} \mathrm{O}$ in methanol (Scheme 3). ${ }^{13}$

In general, it is known that the aziridines are formed via carbene complexes, azomethine ylides or Lewis acid catalyzed intermediates. ${ }^{1 \mathrm{c}}$ Under the present reaction conditions the products formed from dimerization of EDA, i.e., diethyl maleate and fumarate, were not observed. So, the reaction proceeds via Lewis acid catalyzed intermediates.

In summary, chiral aziridines were prepared from chiral $\mathrm{N}$ -

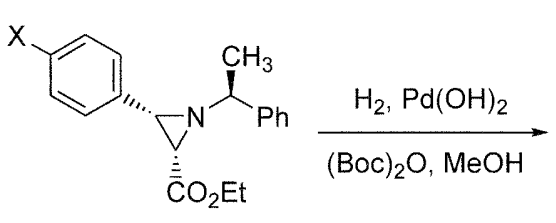

2f: $\mathrm{X}=\mathrm{Cl}$

2h: $\mathrm{X}=\mathrm{NO}_{2}$

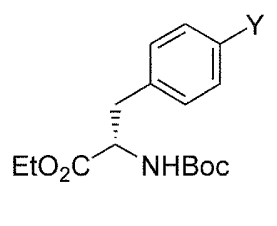

7f: $Y=H$

7h: $\mathrm{Y}=\mathrm{NHBOc}$ benzylimines and ethyl diazoacetate using Co(II) catalyst as Lewis acid in moderate yields. Hydrogenolytic ring opening of the azirines in the presence of $\mathrm{Pd}(\mathrm{OH})_{2}$ and $(\mathrm{Boc})_{2} \mathrm{O}$ afforded the aryalanine derivatives in high yields with retention of stereochemistry.

Acknowledgement. This work was supported by the Brain Korea 21 Project.

\section{References}

1. (a) Osborn, H. M. I.; Sweeney, J. Tetrahedron: Asymmetry 1997, 8, 1693. (b) Tanner, D. Angew. Chem. Int. Ed. Engl. 1994, 33, 599. (c) Jacobsen, E. N. In Comprehensive Asymmetric Catalysis; Jacobsen, E. N.; Pfaltz, A.; Yamamoto, H., Ed.; Springer-Verlag: Berlin, Heidelberg, New York, 1999; Vol. 2, p 607. (d) Atkinson, R. S. Tetrahedron 1999, 55, 1519. (e) Cardillo, G.; Gentilucci, L.; Tolomelli, A. Aldrichimica Acta 2003, 36, 57.

2. Yadav, J. S.; Reddy, B. V. S.; Rao, M. S.; Reddy, P. N. Tetrahedron Lett. 2003, 44, 5275.

3. (a) Antilla, J. C.; Wulff, W. D. J. Am. Chem. Soc. 1999, 121, 5099. (b) Loncaric, C.; Wulff, W. D. Org. Lett. 2001, 3, 3675.

4. Casarrubios, L.; Pérez, J. A.; Brookhart, M.; Templeton, J. L. J. Org. Chem. 1996, 61, 8358.

5. Ha, H.-J.; Kang, K.-H.; Suh, J.-M.; Ahn, Y.-G. Tetrahedron Lett. 1996, 37, 7069 .

6. Sengupta, S.; Mondal, S. Tetrahedron Lett. 2000, 41, 6245.

7. (a) Juhl, K.; Hazell, R. G.; Jprgensen, K. A. J. Chem. Soc. Perkin Trans. 1 1999, 2293. (b) Lee, S.-H.; Han, T.-D.; Yu, K. A.; Ahn, K.-H. Bull. Korean Chem. Soc. 2000, 22, 449.

8. Rasmussen, K. G.; Jprgensen, K. A. J. Chem. Soc., Perkin Trans. 1 $1997,1287$.

9. Mayer, M. F.; Hossain, M. M. J. Org. Chem. 1998, 63, 6839.

10. Kubo, T.; Sakasguchi, S.; Ishii, Y. Chem. Commun. 2000, 625.

11. (a) Nagayama, S.; Kobayashi, S. Chem. Lett. 1998, 685. (b) Xie, W.; Fang, J.; Li, J.; Wang, P. G. Tetrahedron 1999, 55, 12929.

12. The following experimental procedure for the preparation of the aziridine cis-2b is representative: Under nitrogen atmosphere, to a stirred solution of $\mathrm{AgBF}_{4}(0.2 \mathrm{mmol}, 0.4$ equiv) in acetone $(1 \mathrm{~mL})$ was added $\mathrm{CoCl}_{2}(0.1 \mathrm{mmol}, 0.2$ equiv $)$ in acetone $(1 \mathrm{~mL})$ at room temperature. Imine $(0.5 \mathrm{mmol}, 1.0$ equiv) in acetone $(3 \mathrm{~mL})$ and ethyl diazoacetate ( $1.5 \mathrm{mmol}, 3.0$ equiv) were added to a reaction mixture and stirred for $10 \mathrm{~h}$. The reaction mixture was concentrated, dissolved in ether and filtered through a short silica gel column. Evaporation of solvent, followed by flash chromatography (EtOAc: $\mathrm{Hex}=1: 5$ ) allowed separation of the diastereomeric products.

(2S,3S)-3-Phenyl-1-[(S)- $\alpha$-methylbenzyl]aziridine-2-carboxylic acid ethyl ester (2b): $m p$ 91-92 ${ }^{\circ} \mathrm{C} ;[\alpha]_{\mathrm{D}}^{20}=+63.5$ (c 1.0, $\left.\left.\mathrm{CH}_{2} \mathrm{Cl}_{2}\right)\right]$; ${ }^{1} \mathrm{H}$ NMR $\left(400 \mathrm{MHz}, \mathrm{CDCl}_{3}\right) \delta$ 7.46-7.14 (m, 10H), 4.05-3.89 (m, 2H), $2.99(\mathrm{~d}, J=6.8 \mathrm{~Hz}, 1 \mathrm{H}), 2.87(\mathrm{q}, J=6.6 \mathrm{~Hz}$, $1 \mathrm{H}), 2.61(\mathrm{~d}, J=7.0 \mathrm{~Hz}, 1 \mathrm{H}), 1.55(\mathrm{~d}, J=6.6 \mathrm{~Hz}, 3 \mathrm{H}), 0.98(\mathrm{t}, J=$ $7.3 \mathrm{~Hz}, 3 \mathrm{H}) ;{ }^{13} \mathrm{C}$ NMR $\left(100 \mathrm{MHz}, \mathrm{CDCl}_{3}\right) \delta 168.25,143.23$, 135.14, 128.37, 127.74, 127.66, 127.26, 127.17, 126.91, 69.77, 60.65, 47.34, 46.00, 22.92, 13.92; IR (KBr) $1742 \mathrm{~cm}^{-1}$; Mass spectrum, $m / z 295\left(\mathrm{M}^{+}\right), 250,235,190,162,146,117,105,79,77$, 51; Anal. Calcd for $\mathrm{C}_{19} \mathrm{H}_{21} \mathrm{NO}_{2}: \mathrm{C}, 77.26 ; \mathrm{H}, 7.17 ; \mathrm{N}, 4.74$. Found: C, 77.10; H, 7.17; N, 4.76.

13. Chang, J.-W.; Bae, J. H.; Shin, S.-H.; Park, C. S.; Choi, D.; Lee, W. K. Tetrahedron Lett. 1998, 39, 9193.

Scheme 3 